\title{
Laparoscopic percutaneous internal ring suturing for paediatric inguinal hernias: a South African tertiary centre experience
}

\author{
S Madziba, (i) S Harilal, (D) H Mangray (iD) \\ Department of Paediatric Surgery, Grey's Hospital, South Africa
}

Corresponding author, email: mangrayh@gmail.com

Background: Laparoscopic inguinal hernia repair is routine in many paediatric centres. One of several techniques described in 2006 is the laparoscopic percutaneous internal ring suturing (PIRS) technique. This technique which is not widely used in South Africa has been adopted for paediatric inguinal hernias at our institution, and this is a review of the experience.

Method: The study is a retrospective review of the patients who underwent PIRS (a single port combined percutaneous procedure used to close the internal ring) at our hospital from October 2018 until March 2020. We describe the technique in our setting and review our cohort of patients, demographics, clinical presentation, operative duration and complications. Results: One hundred and ten laparoscopic PIRS procedures were performed on 94 patients; 15 had bilateral inguinal hernias. Polydioxanone and Prolene were the sutures used for the procedures. Average operative time was 12 minutes for unilateral hernias and 33 minutes for bilateral hernias. All operations were completed laparoscopically. Follow-up was for a minimum of three months, but up to 21 months. Complications included three intraoperative haematomas, three hydrocoeles and two umbilical granulomas postoperatively. There were two recurrent hernias of which one was managed with redo PIRS; the other required open herniotomy for an irreducible obstructed hernia.

Conclusion: This review is supportive evidence that the PIRS technique for managing paediatric inguinal hernias in a tertiary institution in South Africa can be performed safely with few complications.

Keywords: inguinal hernia, paediatric, laparoscopy, percutaneous internal ring suturing

Supplementary video available online: http://sajs.redbricklibrary.com/index.php/sajs/article/view/3498

\section{Introduction}

Paediatric inguinal hernias are one of the most prevalent conditions requiring surgical intervention. The conventional surgical approach is usually an open procedure via an inguinal incision. Inguinal herniotomy involves the separation of the patent processus vaginalis (PPV) from the vas and vessels, division of the sac, and subsequent ligation of it. Advances in paediatric surgery have developed to a point where inguinal herniotomies are routinely performed laparoscopically in many centres internationally., ${ }^{1,2}$ The benefits of paediatric laparoscopic inguinal hernia repair over the open procedure include direct visualisation of the internal inguinal ring without dissection of the inguinal canal, confirmation of a PPV in suspected cases; it allows assessment of the contralateral inguinal ring, results in fewer episodes of haematomas and vas injury, is associated with improved pain and cosmesis and has lower recurrence rates. ${ }^{3}$ There have been various laparoscopic approaches described for inguinal herniotomies with the use of two or three ports. ${ }^{4-6}$ Professor Patkowski developed the laparoscopic percutaneous internal ring suturing (PIRS) technique for the management of paediatric inguinal hernias in $2006 .^{7}$ PIRS involves the use of a single umbilical camera port of 3-5 mm combined with the percutaneous insertion of a suture, using a needle, around the PPV and tied externally to close the internal ring. The PIRS technique has been confirmed in numerous publications to be reproducible and associated with few complications. ${ }^{8-11}$ We have adopted the PIRS technique for paediatric patients at our institution since 2018, and we reviewed our experience since initiation.

\section{Methods}

This retrospective observational study was conducted using the STROBE guidelines. ${ }^{12}$ This study was granted ethical approval by the Biomedical and Research Council of UKZN. This study was a review of all paediatric patients above the age of six months who presented to our institution with inguinal hernias and had the PIRS procedure. The study was conducted in a tertiary level referral hospital that provides paediatric surgical services for area two of the KwaZuluNatal province. The decision to perform the procedure on patients above six months of age was made to allow for open herniotomies to be done in younger patients for teaching purposes. The data were collected retrospectively from October 2018 until June 2020 and collated on an Excel document. Qualified paediatric surgeons performed all operations. Patients were followed up for a minimum of three months. 

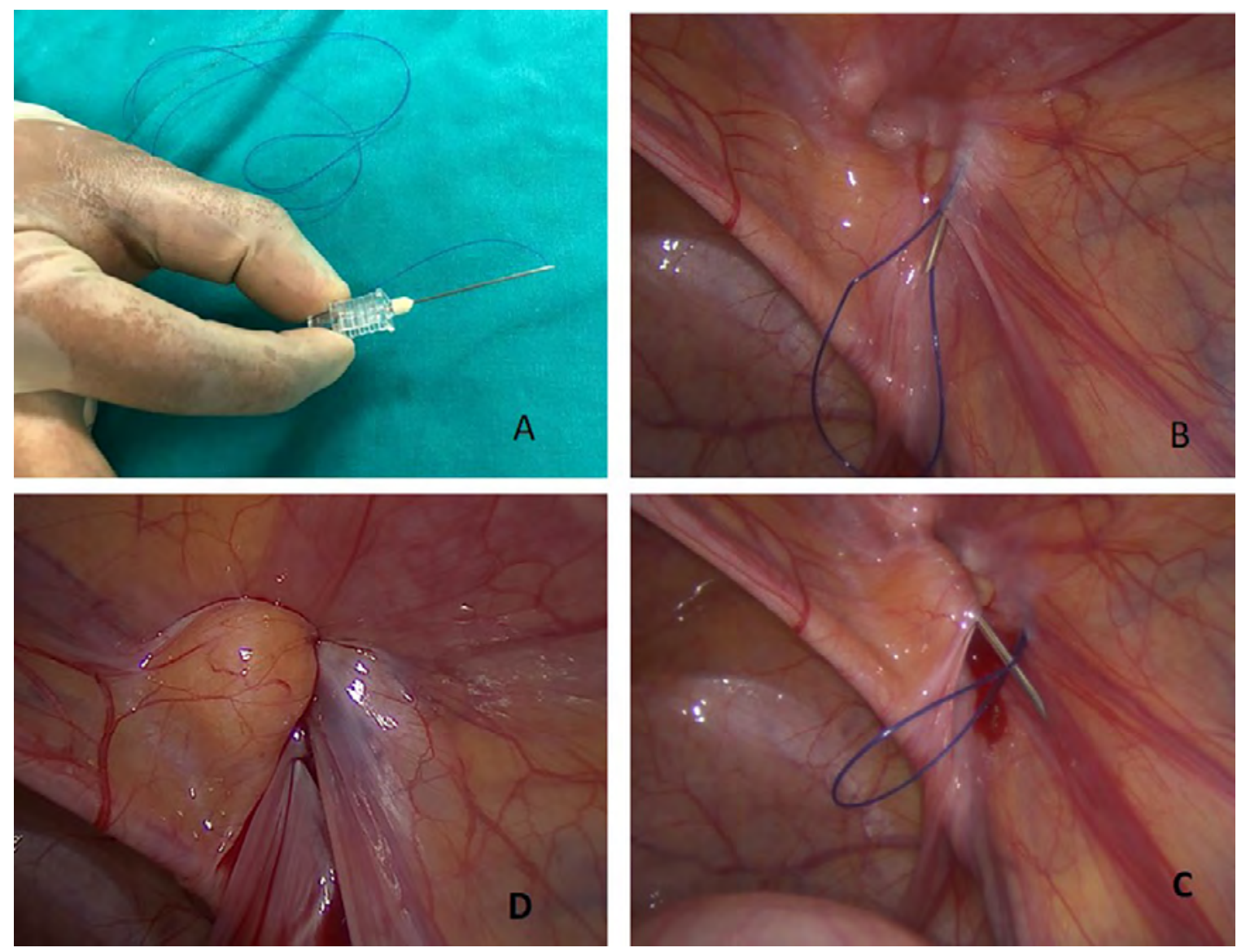

Figure 1: PIRS procedure outlined from $A$ to $D$

The procedure was performed under a general anaesthetic without regional caudal anaesthesia. After emptying the bladder, a single $5 \mathrm{~mm}$ umbilical port was inserted using the open technique. The carbon dioxide insufflation pressures varied between 8-12 $\mathrm{mmHg}$ depending on the age of the child. The patient was then placed in a Trendelenburg position to allow the small bowel to move away from the pelvis and improve visualisation of the operative field. A 30-degree lens was used with some lateral rotation to allow easier visualisation of the internal inguinal rings. Both the right and left internal inguinal rings were then assessed, and the PPV was confirmed. A single stab of the skin was made directly over the internal ring of the affected side.

The needle and proximal curve of the
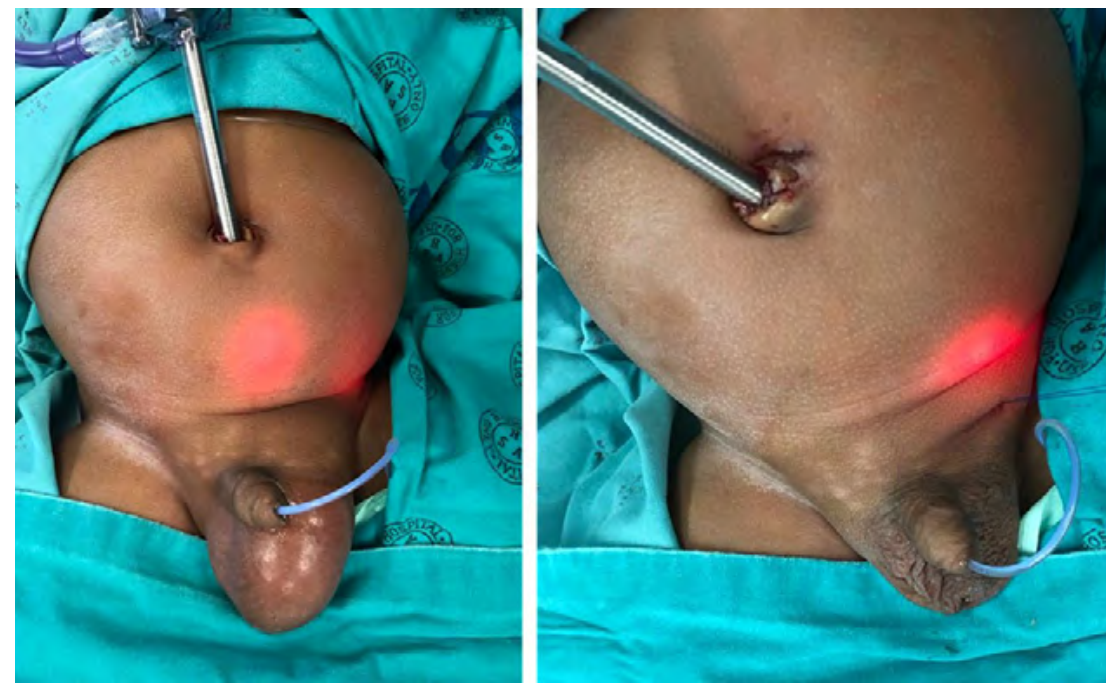

Figure 2: Pneumoscrotum does not recur post PIRS as indicated by image on right suture were removed so that a straight suture remained for ease of passage. An 18-gauge spinal introducer needle was flushed with saline before the suture is passed through it and brought out the tip of the needle to create a loop. The needle and looped suture are introduced via the skin stab and passed under laparoscopic visualisation around the lateral wall peritoneum of the internal ring (Figure 1). The loop was pushed further into the abdominal cavity. Care was taken to avoid the vas and vessels. The needle was then removed while retaining the loop within the abdominal cavity. The needle was then passed via the

medial wall of the internal ring, and the distal end of the suture was passed into the abdomen and through the initial loop that was within the abdomen. The needle was removed, and the initial loop is used to withdraw the straight end to create a purse-string suture around the internal ring, which is tied externally. The hernia can now also be confirmed to be closed and airtight (Figure 2). The pneumo-peritoneum was decompressed, the port site was closed with an absorbable suture, and Steristrips used for the skin. Lignocaine was injected as a local anaesthetic for the port and puncture sites. 
All patients were reviewed clinically at seven days and one month following the operation. Parents were advised to return if there was any evidence of recurrence or problems with the surgical wound sites. Follow-up was until June 2020 , and each patient had a minimum of three months' follow-up.

Information collected included the patients' age, sex, the side of the inguinal hernia, the type of presentation, data regarding the operative duration, type of suture material used, and intra- and postoperative complications. The operative duration was defined as from skin incision to skin closure. All data were collected using a data sheet and annotated on an Excel document.

\section{Results}

Ninety-four patients had 110 laparoscopic PIRS procedures performed with 15 patients having bilateral PPV, and two had recurrent hernias. There were 90 male patients and four female patients who presented between 6 months and 12 years of age. Patients presented for elective surgery in 90 cases, and there were four irreducible inguinal hernias. One patient was treated for a recurrence of a previous open inguinal herniotomy. The predominant side for the unilateral inguinal hernias was the right in 55 patients and the left in 23 patients. In four patients, suspected inguinal hernias were excluded on laparoscopy, while in five patients, we confirmed a contralateral PPV (Diagram 1). The operative time was an average of 12 minutes (7-25 minutes) for unilateral hernias and 33 minutes (25-45 minutes) for bilateral hernias. The omentum in one case was reduced successfully with the lens; however, the bladder was found in another case, and an additional $3 \mathrm{~mm}$ port and grasper were required to reduce it. Sixty-one cases were performed using Polydioxanone 3.0, and 49 cases were performed using Prolene 3.0. All procedures were completed laparoscopically.

The complications experienced were classified as intraoperative and postoperative (Table I). During the operation, we experienced three haematomas, two as a result of injury to the inferior epigastric vessels and one of the testicular vessels. All were managed conservatively with no further issues. Postoperative complications included two umbilical granulomas that occurred in patients, one patient represented with umbilical pain that was later attributed to constipation, and he recovered after a fleet enema. Hydrocoeles were found in three patients a month after PIRS of whom one had a re-laparoscopy, and the PPV was confirmed to be closed. The three hydrocoeles were aspirated to dryness, and none has recurred after a minimum of eight months. We had two recurrent hernias; one had the repair done with Polydioxanone 3.0 and presented three months after the PIRS. This patient had a redo PIRS, and the processus vaginalis was patent with no suture identified. The second patient had the PIRS done with Prolene 3.0 and presented five months post PIRS with an irreducible obstructed hernia of the same side. He had a laparoscopic approach initially but as the bowel was too oedematous to reduce safely, it was converted to an open reduction and herniotomy. The Prolene suture was not identified at the re-operation, and the patient had recovered with no further recurrence to date.

\section{Discussion}

Paediatric inguinal hernia repair remains a common surgical procedure most often performed as a standard open

\begin{tabular}{lcc}
\hline Table I & & \\
\hline Complication & Number of cases & Management \\
\hline Intraoperative & & \\
\hline Haematoma & 3 & Conservative \\
Conversion to open & 0 & \\
Postoperative & & \\
\hline Umbilical granuloma & 2 & Silver nitrate \\
Hydrocoele & 3 & Aspirated \\
Recurrence & 2 & Surgery
\end{tabular}

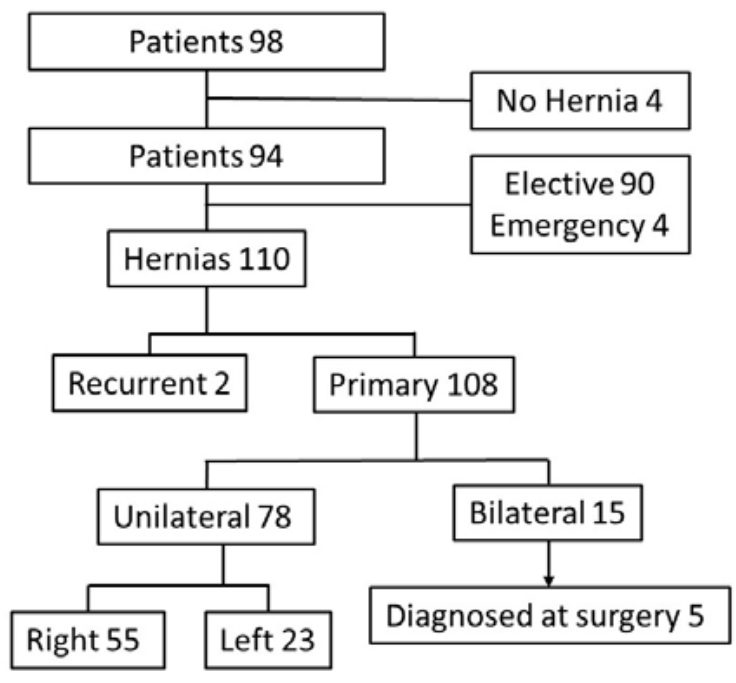

Diagram 1: Flow chart of patients managed with PIRS

procedure. The open herniotomy involves dissection of the cord structures, which includes division of the cremaster, separation of the vas and vessels from the sac and division, and ligation of the sac. This dissection contributes to tissue trauma, bleeding, oedema and haematomas of the scrotum, and predisposes the vas and vessels to injury. ${ }^{13-16}$ Laparoscopic inguinal hernia repair has proven to have benefits such as fewer wound infections, lesser pain, the ability to diagnose a contralateral PPV and improved cosmesis over the open procedure. , $^{7,10,17}$

The PIRS technique uses a single port and is effective, as demonstrated in many studies..$^{7-10}$ The use of a single port is the standard technique, and we were able to complete the majority of procedures using this technique. In this series, we had one case that required the use of an additional port for a grasper to be inserted to reduce the bladder. This is, however, a unique situation with the bladder prolapsing into the PPV. The use of an additional port has been advocated, not only if required for reduction, but also to induce scarring of the PPV edges. ${ }^{3}$ The scarring was previously shown to improve closure of the PPV in a rabbit model. ${ }^{18}$ This may be true; however, our short-term recurrence rates remain low despite no scarring.

If we consider the changes that occur after ligation of a PPV, we should achieve closure following ischaemia around the ligature site and scarring as a result of the healing process. This poses the question as to whether scarring is necessary as it is not part of an open herniotomy either. Another consideration is hydro-dissection around the PPV, where saline is used to elevate the peritoneum off the 
abdominal wall and reduce injury to vas and vessels during the procedure. ${ }^{3}$

The benefit of laparoscopy is so that we can confirm the diagnosis and also exclude a contralateral PPV. ${ }^{3,10} \mathrm{We}$ were able to avoid additional surgery in four cases where the processus vaginalis was confirmed to be closed on laparoscopy and in the five cases where the contralateral PPV was closed at the same operation. We had three haematomas during the procedure, and one involved the testicular vessels. Haematomas have been described in other series, and our incidence remains low (2.7\%) despite no hydro-dissection. They are usually contained within the extra-peritoneal space and are managed conservatively with no consequence. ${ }^{7}$

We found that removal of the curve of the suture together with the use of a saline flush through the needle assisted with the easier passage of the suture through the needle. Our recurrence rate was $1.8 \%$, which was in keeping with other published data. $7,9,10$ With regards to recurrence prevention using PIRS, Dvorakevyc et al. looked at performing a double loop suture and found that there were no recurrences compared to the standard single PIRS; however, patients had more postoperative pain. ${ }^{19}$

There were three cases of hydrocoeles post PIRS, and all occurred in older children. Hydrocoele as a complication occurred more often post open herniotomy as compared to laparoscopic herniotomy in the review by Zhang et al. ${ }^{20}$ They were all managed by a single aspiration episode, and none has recurred to date.

An additional technical point was a confirmation during the procedure that the hernia closure was airtight. We were able to monitor the reduced pneumo-scrotum following the closure of the PPV as confirmation of closure. Other concerns that have been raised in the literature were regarding blood flow to the testicle following PIRS. The impact of PIRS on testicular artery blood flow was assessed by Oral et al., and they showed there was no compromise following the procedure. $^{21}$

PIRS being a laparoscopic procedure does have a downside, and one of the issues is the learning curve of the procedure. Barroso et al. looked at the learning curve for PIRS and found that after 35 cases, the complication and conversion rates averaged normal levels. ${ }^{22}$

In all cases, the laparoscopic port sites were closed using Polydioxanone 4.0 suture. Despite using an absorbable suture, two patients developed umbilical granulomas that were managed with silver nitrate application. Stitch site granulomas do occur as a result of the suture material used; however, absorbable sutures are associated with a lower incidence. $^{23}$

If we compare the complications related to laparoscopic PIRS versus open inguinal hernia repairs, we do note a different spectrum of complications with PIRS. Esposito et al. showed higher rates of wound infections, hydrocoeles, iatrogenic cryptorchidism, and testicular atrophy with open herniotomies. ${ }^{24}$ Comparatively, we had no wound sepsis, testicular atrophy or cryptorchidism and only three hydrocoeles (Table I). Despite the few intraoperative issues, we were able to complete all cases laparoscopically.

The limitations of this review include the fact that it is a retrospective review. We do not have any experience of laparoscopic PIRS in children younger than six months of age; however, according to the literature, it has been successfully done in neonates as well. ${ }^{7}$ We have not presented our open herniotomy experience and will review this in the future to compare our outcomes. Recurrences most often occur between 2-6 months, and since we limited our current review to 3 months post-surgery, there is still a possibility of recurrences in the more recent cases. ${ }^{7,10} \mathrm{We}$ acknowledge that there was no randomisation of the use of Polydioxanone and Prolene sutures.

\section{Conclusion}

Laparoscopic inguinal hernia repair has clear benefits and presents with a different spectrum of complications related to the procedure. These complications can be managed non-surgically in most instances and do not seem to pose a serious problem. This review is supportive evidence that the PIRS technique for managing paediatric inguinal hernias is simple and can be safely performed in a tertiary institution in South Africa without the use of additional interventions such as hydro-dissection and scarring.

\section{Conflict of interest}

The authors declare no conflict of interest.

\section{Funding source}

No funding was required.

\section{Ethical approval}

This study was granted ethical approval by the Biomedical and Research Council of University of KwaZulu-Natal.

\section{ORCID}

S Madziba (iD) https://orcid.org/0000-0003-4077-3465

S Harilal iD https://orcid.org/0000-0003-1909-4652

H Mangray (iD https://orcid.org/0000-0002-5204-3542

\section{REFERENCES}

1. Zani A, Eaton S, Hoellwarth M, et al. Management of pediatric inguinal hernias in the era of laparoscopy - results of an international survey. J Pediatr Surg. 2014;24:9-13. https:// doi.org/10.1055/s-0033-1354586.

2. Schier F, Montupet P, Esposito C. Laparoscopic inguinal herniorrhaphy in children - a three-centre experience with 933 repairs. J Pediatr Surg. 2002;37:395-7. https://doi. org/10.1053/jpsu.2002.30842.

3. Ponsky TA, Nalugo M, Ostlie DJ. Pediatric laparoscopic inguinal hernia repair - a review of the current evidence. J Laparoendosc Adv Surg Tech. 2014;24:183-7. https://doi. org/10.1089/lap.2014.9998.

4. Montupet P, Esposito C. Laparoscopic treatment of congenital inguinal hernia in children. J Pediatr Surg. 1999;34:420-3. https://doi.org/10.1016/S0022-3468(99)90490-6.

5. Parelkar SV, Oak S, Gupta R, et al. Laparoscopic inguinal hernia repair in the pediatric age group - experience with 437 children. J Pediatr Surg. 2010;45:789-92. https://doi. org/10.1016/j.jpedsurg.2009.08.007.

6. Gorsler CM, Schier F. Laparoscopic herniorrhaphy in children. Surg Endosc Interv Tech. 2003;17:571-3. https://doi. org/10.1007/s00464-002-8947-y.

7. Patkowski D, Czernik J, Chrzan R, Jaworski W, Apoznański W. Percutaneous internal ring suturing: a simple minimally invasive technique for inguinal hernia repair in children. J Laparoendosc Adv Surg Tech. 2006;16:513-7. https://doi. org/10.1089/lap.2006.16.513. 
8. Erginel B, Akin M, Yildiz A, et al. Percutaneous internal ring suturing as a first choice laparoscopic inguinal hernia repair method in girls - a single-centre study in 148 patients. Pediatr Surg Int. 2016;32:697-700. https://doi.org/10.1007/s00383016-3903-4.

9. Wolak PK, Patkowski D. Laparoscopic inguinal hernia repair in children using the percutaneous internal ring suturing technique - own experience. Videosurgery Miniinvasive Tech. 2014;1:53-8. https://doi.org/10.5114/wiitm.2014.40389.

10. Thomas DT, Göcmen KB, Tulgar S, Boga I. Percutaneous internal ring suturing is a safe and effective method for the minimal invasive treatment of pediatric inguinal hernia experience with 250 cases. J Pediatr Surg. 2016;51:1330-5. https://doi.org/10.1016/j.jpedsurg.2015.11.024.

11. Baltrak YA. Results of inguinal hernia repair by PIRS method in girls. Eurasian J Med Investig. 2018;2(3):148-51. https:// doi.org/10.14744/ejmi.2018.27146.

12. Von Elm E, Altman DG, Egger M, et al. The strengthening the reporting of observational studies in epidemiology (STROBE) statement - guidelines for reporting observational studies. Lancet. 2007;370:1453-7. https://doi.org/10.1016/ S0140-6736(07)61602-X.

13. Nazem M, Dastgerdi MMH, Sirousfard M. Outcomes of pediatric inguinal hernia repair with or without opening the external oblique muscle fascia. J Res Med Sci Off J Isfahan Univ Med Sci. 2015;20:1172-6. https://doi.org/10.4103/17351995.172985.

14. Askarpour S, Peyvasteh M, Javaherizadeh H, Mehdianzadeh F. Recurrence and complications of pediatric inguinal hernia repair over 5 years. Ann Pediatr Surg. 2013;9:58-60. https:// doi.org/10.1097/01.XPS.0000426350.23987.7a.

15. Taqvi SRH, Akhtar J, Batool T, Tabassum R, Mirza F. Complications of inguinal hernia surgery in children. J Coll Physicians Surg Pak. 2006;16:532-5. https://doi.org/8.2006/ JCPSP. 532535

16. Erdoğan D, Karaman İ, Aslan MK, Karaman A, Çavuşoğlu YH. Analysis of 3776 pediatric inguinal hernia and hydrocele cases in a tertiary centre. J Pediatr Surg. 2013;48:1767-72. https://doi.org/10.1016/j.jpedsurg.2012.09.048.

17. Yang $\mathrm{C}, \mathrm{Zhang} \mathrm{H}, \mathrm{PuJ}$, etal.Laparoscopic vsopenherniorrhaphy in the management of pediatric inguinal hernia - a systemic review and meta-analysis. J Pediatr Surg. 2011;46:1824-34. https://doi.org/10.1016/j.jpedsurg.2011.04.001.

18. Blatnik JA, Harth KC, Krpata DM, et al. Stitch versus scarevaluation of laparoscopic pediatric inguinal hernia repair: a pilot study in a rabbit model. J Laparoendosc Adv Surg Tech. 2012;22:848-51. https://doi.org/10.1089/lap.2012.0137.

19. Dvorakevych AO, Pereyaslov AA, Tkachyshyn YI. Modification of the traditional percutaneous internal inguinal ring suturing (PIRS method). Paedriatric Surgery. 2017;2(55):57-61.

20. Zhang Y, Chao M, Zhang X, et al. Does the laparoscopic treatment of paediatric hydroceles represent a better alternative to the traditional open repair technique? A retrospective study of 1332 surgeries performed at two centres in China. Hernia. 2018;22:661-9. https://doi.org/10.1007/s10029-017-1715-7.

21. Oral A, Karaca L, Ahiskalioglu A, et al. Effects of laparoscopic hernia repair by PIRS (percutan internal ring suturing) technique on testicular artery blood supply. J Investig Surg Off J Acad Surg Res. 2019;32:343-7. https://doi.org/10.1080/ 08941939.2017.1419317.

22. Barroso C, Etlinger P, Alves AL, et al. Learning curves for laparoscopic repair of inguinal hernia and communicating hydrocele in children. Front Pediatr. 2017;5:207. https://doi. org/10.3389/fped.2017.00207.

23. Nagar H. Stitch granulomas following inguinal herniotomy a 10-year review. J Pediatr Surg. 1993;28:1505-7. https://doi. org/10.1016/0022-3468(93)90442.

24. Esposito C, St Peter SD, Escolino M, et al. Laparoscopic versus open inguinal hernia repair in pediatric patients: a systematic review. J Laparoendosc Adv Surg Tech. 2014;24:811-8. https://doi.org/10.1089/lap.2014.0194. 